\title{
HUNDERT JAHRE MCLUHAN
}

von SVEN GRAMPP

W. Terrence Gordon, McLuhan: A Guide for the Perplexed, New York, London (Continuum Inter. Publis.) 20 Iо.

Paul Grossweiler (Hg.), Transforming McLuhan. Cultural, Critical And Postmodern Perspectives, New York u. a. (Peter Lang) 2010.

Christian Huberts, Raumtemperatur. Marshall McLuhans Kategorien <heiß> und <kalt> im Computerspiel, Salzhemmendorf (blumenkamp) 20 Io.

Derrick de Kerckhove, Martina Leeker, Kerstin Schmidt (Hg.), McLuhan neu lesen. Kritische Analysen im 2I. Fabrhundert, Bielefeld (Transcript) 2008.

Robert K. Logan, Understanding New Media. Extending Marshall McLuhan, New York u. a. (Peter Lang) 20 Io.

Marshall McLuhan, The Classical Trivium: The Place of Thomas Nashe in the Learning of His Time, hg. von W. Terrence Gordon, Berkeley (Ginko) 2006.

Marshall McLuhan starb in der Silvesternacht 1980. Sein Tod ereignete sich damit zu Beginn einer Dekade, an deren Ende er als Gründungsvater der Medienwissenschaft, hierzulande zumindest, eine ungeahnte Renaissance erleben durfte. Doch auch das ist schon eine Weile her. Die deutschsprachigen Ausgaben seiner Hauptwerke Die Gutenberg-Galaxis und Die magischen Kanäle finden sich heute nur noch in manchen Institutsbibliotheken und Antiquariaten. Nun, 2011, jährt sich McLuhans Geburtstag zum hundertsten Mal. Mit diversen Veranstaltungen unter anderem in Toronto, Berlin, Rom und vor allem einer hochrangig besetzten internationalen Tagung in Barcelona wird das gefeiert. ${ }^{1}$ Unter dem Motto «McLuhan Ga- laxy. Understanding Media, Today» soll der kanadische Forscher damit wieder in Erinnerung gebracht werden. Auch publizistisch warfen hundert Jahre McLuhan Schatten voraus. Vor allem im letzten Jahr sind auffallend viele Publikationen zu McLuhan erschienen. Noch einige mehr sind für 2011 zu erwarten. ${ }^{2}$ In den Veranstaltungen und Publikationen geht es zumeist nicht nur darum, wie Medien (today zu verstehen sind, sondern vor allem auch ob und, wenn ja, in welcher Weise Medien mit McLuhan überhaupt (noch) angemessen zu verstehen sind. An der Beantwortung dieser Frage scheiden sich die Geister, auch in den hier zu rezensierenden Büchern, zum Teil quer durch die Bände selbst.

Bevor ich jedoch näher auf einige dieser Lektüren an der Schwelle zu McLuhans hundertstem Geburtstag eingehe, scheint es durchaus ratsam, kurz zu skizzieren, welchen Stellenwert McLuhan zumindest in der deutschsprachigen Medienwissenschaft eigentlich hat oder zumindest lange Zeit hatte. So lässt sich besser verstehen, was es denn bedeuten könnte, «McLuhan neu» zu «lesen» (wie es eines der zu rezensierenden Bücher im Titel verspricht). Seit den 196oer Jahren ist McLuhan ein durchaus umstrittener Medien- und Kulturtheoretiker. In der deutschsprachigen Forschungslandschaft spielte er insbesondere für die sich in den 1970er und 1980er Jahren konstituierende Medienwissenschaft indes nicht nur die Rolle des Buhmanns. ${ }^{3}$ Vielmehr erhielt McLuhan eine zentrale Rolle als Gewährsmann für einen spezifischen Zugriff auf Kultur, Gesellschaft und Medien und wurde retrospektiv als Gründungsfigur medienwissenschaftlicher Forschung inthronisiert. ${ }^{4}$ Und auch wenn er nicht gleich als ein «Diskursivitätsbegründen im Sinne Michel Foucaults eingesetzt wird, dann doch zumindest als derjenige, der den entscheiden- 
den und bis heute einzig konsensfähigen Schlachtruf der Medienwissenschaft formuliert hat, nämlich The Medium is the Message.

Diese Einschätzung ist spätestens mit dem Jahrtausendwechsel auch bis in die «Basisliteratun durchgesickert und bildet seither kanonisches Wissen. Beispielsweise heißt es in Rainer Leschkes Einführung in die Medientheorie von 2003: «Mit McLuhans These, dass die Medien selbst die Botschaft bildeten, und mit dem dadurch evozierten Übergang des Erkenntnisinteresses auf die Form von Medien ist allerdings von McLuhan erst das Terrain für eine eigenständige Medienwissenschaft geschaffen worden.» ${ }^{5}$ Und Jochen Hörisch schreibt in seiner zwei Jahre früher zum ersten Mal erschienenen Geschichte der Medien gar, McLuhans These habe es der Medientheorie überhaupt erst möglich gemacht, zur «diensthabenden Fundamentaltheorie» ${ }^{6}$ aufzusteigen.

Bereits in den 1980er Jahren erhob Friedrich Kittler, dessen materialistische Medienarchäologie selbst inzwischen international als Inbegriff einer German Media Theory figuriert, ${ }^{7}$ McLuhan zum «Diskursivitätsbegründen einer spezifischen Medienwissenschaft. McLuhan wurde dabei gegen philologisch orientierte Medieninhaltsanalysen und soziologische Kommunikationsforschung und für eine medienmaterialistische Position wissenschaftstheoretisch und -politisch in Stellung gebracht. ${ }^{8}$ In einer Vorlesung Kittlers heißt es zur Vorreiterrolle McLuhans rückblickend: Ohne dessen «berühmte[...] Formel» vom Medium, das die Botschaft ist, «die es nachgerade verbietet, hinter technisch erzeugten Oberflächen noch etwas anderes zu suchen», hätte sich die Medienwissenschaft «nicht als solche» entwickeln können. ${ }^{9}$ Kittler betrachtet McLuhan aber durchaus kritisch. So wirft er ihm etwa vor, einen Rest humanwissenschaftlichen Idealismus nicht über Bord geworfen zu haben und immer noch zu glauben, Medien ließen sich hermeneutisch verstehen. So heißt es sehr deutlich in Grammphon Film Typewriter: «Medien zu verstehen, bleibt - trotz «Understanding Media` im Buchtitel McLuhans - eine Unmöglichkeit, weil gerade umgekehrt die jeweils herrschenden Nachrichtentechniken alles Verstehen fernsteuern.» ${ }^{\mathbf{1 0}}$ Ebenso kritisch äußert sich Kittler hinsichtlich McLuhans heilsgeschichtlich aufgeladener Hoffnung einer globalen Gesellschaft: Hier verwechsele der bereits in den 1930er Jahren zum Katholizismus konvertierte Medienforscher schlicht den «Heiligen Geist und Turings Maschine»." So ist denn aus Kittlers Perspektive McLuhan vor allem für digitale Medientechnologie nicht mehr zuständig. Diese Einschätzung wurde im weiteren Verlauf des medienwissenschaftlichen Diskurses ein topischer Kritikpunkt, der sich ebenfalls in der jüngsten Literatur zu McLuhan wiederfindet. ${ }^{12}$

Ein anderer, bis dato nicht minder virulenter Kritikpunkt kommt aus einer ganz anderen Richtung: McLuhan wird spätestens seit dem Erscheinen von Die magischen Kanäle wiederholt vorgeworfen, er vernachlässige soziokulturelle und ökonomische Aspekte bei seiner medientechnisch orientierten Kulturgeschichte ${ }^{13}$ und tendiere damit zum Mediendeterminismus. Ging es Kittler, wie obiges Zitat belegt, bei McLuhan noch zu wenig mediendeterministisch zu, so gilt er anderen als Vorreiter einer Forschungsposition, die fataler Weise Medien als alleinige Ursache für gesellschaftliche und kognitive Prozesse verstehen will und so blind für alle anderen Faktoren bleibt. Eine komplexe medienkulturelle Lage ließe sich so jedenfalls nicht angemessen beschreiben. ${ }^{14}$

Seit mindestens drei Dekaden zirkulieren die angeführten Einschätzungen in der Medienwissenschaft. Wie unterschiedlich auch die jeweiligen Positionen sein mögen, so gut wie immer lassen sie sich doch zumindest innerhalb des geschilderten argumentativen Dreieckgestirns verorten: (1) «McLuhan stellt visionär (Medien-)Wissenschaft auf die Untersuchung medialer Formen um> und/oder: (2) 〈McLuhan hat keine Ahnung von (Computer-)Technologie〉 und/oder: (3) «McLuhan vertritt einen unterkomplexen Mediendeterminismus». Diese Einschätzungen finden sich mehr oder weniger explizit auch in den «Re-Lektüren〉 für das «21. Jahrhundert` wieder, wenngleich sie dort neue Akzentuierungen erhalten und Mischungsverhältnisse unterschiedlicher Art eingehen.

Dementsprechend geben die hier zu rezensierenden Publikationen verschiedene Antworten auf die Frage, ob mit McLuhan Medien (noch) angemessen zu verstehen sind. Dennoch lassen sich grob drei Fraktionen unterscheiden. Die erste Fraktion bejaht die Frage, und zwar enthusiastisch: Mit McLuhan ließen sich immer noch, ja vielleicht sogar besser denn je, Medien verstehen. Der kanadische Forscher, dessen Hauptaugenmerk noch der analogen Welt des Radios und des Fernsehens galt, wird als Visionär eines digitalen Computerzeitalters beschrieben, dem auch im neuen Millenium zu folgen sei. Diese Fraktion nenne ich deshalb die Affirmierer. Die zweite Fraktion verneint im Gegensatz dazu die Frage: McLuhan habe uns, so deren Position, über die Medien nichts (mehr) zu sagen. Wenn überhaupt gebe vielmehr die medienkultu- 
relle Konstellation der 196oer und 1970er Jahre Aufschluss über McLuhan und die mit ihm assoziierten Akteure. Die Ideen des «High Priest of Popcult and Metaphysican of Media», ${ }^{15}$ wie ihn der Playboy 1969 bezeichnete, werden also nicht für die Gegenwart aktualisiert oder gar in die Zukunft fortgeschrieben, sondern, genau umgekehrt, historisiert und diskursiv kontextualisiert. Vertreter dieser Sicht der Dinge lassen sich demgemäß als die Diskursivierer bezeichnen. Eine dritte, recht kleine Fraktion entkoppelt McLuhan von jeglicher Anbindung an medienkulturelle Entwicklungen sowie von Fragen nach der vermeintlichen medialen Spezifik der Gegenwart. Mitglieder dieser Fraktion behaupten: Nicht Medien zu verstehen war McLuhans Ziel, sondern, angefangen von seiner Dissertation über den englischen Satiriker Thomas Nashe und das antike Trivium bis zum posthum veröffentlichten Laws of Media, habe er fieberhaft an einer universellen, medien-, ja buchstäblich weltübergreifenden Grammatologie gearbeitet. Anhänger dieser These sollen deshalb den Namen die Grammatisierer tragen.

Zur Fraktion der Affirmierer gehört sicherlich Robert K. Logan, der 1968 Professor für Physik an der Universität in Toronto wurde und dort zusammen mit McLuhan an einigen Projekten arbeitete. Schon der Titel des mit knapp 400 eng bedruckten Seiten recht umfangreichen Buches signalisiert deutlich, dass sich hier jemand in die Traditionslinie McLuhans stellt, ja es sich dabei um eine Apologie der Medienforschung McLuhan'scher Provenienz handeln muss: Understanding New Media. Extending Marshall McLuhan. «Extending, bedeutet hier vor allem: McLuhans Ansatz wird auf (New Media) ausgeweitet, die McLuhan selbst noch nicht beschreiben konnte, die aber in seinem Sinn zu beschreiben seien. Es geht unter vielen, sehr vielen anderen Medien, die mit enzyklopädischem Fleiß abgearbeitet werden, dementsprechend beispielsweise um Scanner, Blogs oder auch um Fernsehen auf dem iPod.

Christian Huberts ist zwar sicherlich auch der Fraktion der Affirmierer zuzuordnen, die McLuhans Thesen zur Beschreibung digitaler Gegenwart nutzen. Er will aber nicht wie Logan gleich alle neuen Medien mit McLuhan verstehen, sondern nur das Computerspiel. Zudem möchte Huberts dazu nicht den ‘gesamten` McLuhan nutzen, sondern nur dessen metaphorische Gegenüberstellung von heißen und kalten Medien, die dieser an prominenter Stelle in Die magischen Kanäle zur Unterscheidung partizipatorischer Wirkungen von Medien eingeführt hat. Nach McLuhan stimulieren heiße Medien einen Sinn, sind detailreich und verlangen nur ein «geringes Maß an persönlicher Beteiligung». ${ }^{16}$ Dagegen regen kalte Medien mehrere Sinne an, sind dabei detailarm und verlangen dementsprechend ein hohes Maß an persönlicher Beteiligung. Huberts tut gut daran, dieser Gegenüberstellung gleich zu Beginn seines Buches den essentialistischen Zahn zu ziehen, der einen bei McLuhans Beschreibungen durchaus schmerzen kann. Nach der geglückten Operation heißt es dann: Es gebe keine Medien, die per se warm oder kalt seien, sondern immer nur Relationen und Mischungsverhältnisse. Anhand exemplarischer Analysen einiger Computerspiele kommt Huberts auf diesem Wege zu einem durchaus überzeugenden, weil variablen Modell «zur Beschreibung der partizipatorischen Wirkung von Medien» (S. 11) und liefert damit, seiner eigenen Zielsetzung entsprechend, tatsächlich eine «Sensibilisierung für die unterschiedlichen Temperierungen von Spielelementen» (S. 177).

Hier zeigt sich denn auch schön der mitunter sehr unterschiedliche Umgang der Affirmierer mit McLuhan. So geht es bei Logan um das Festhalten an den vermeintlich zentralen Grundaxiomen McLuhans, mit erheblichem Auf-

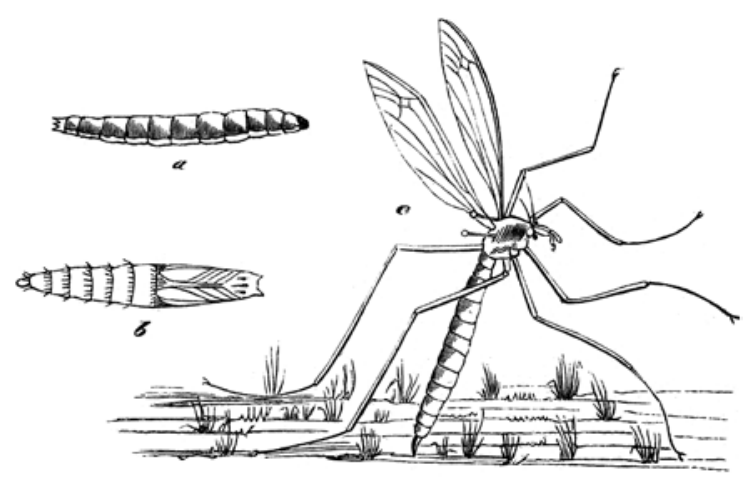

wand und Willen zur Systematisierung. Das ist zumindest um den Preis erkauft, McLuhans Ausführungen auf einige wenige Thesen zuzustutzen und zumindest eines seiner Grundaxiome zu unterschlagen. McLuhan selbst hat sich nämlich einer systematischen Herangehensweise immer verweigert. Wilde Assoziationsketten und kühne Analogien, elliptische, sprunghafte Darstellungsform, nicht zuletzt ein Metaphernreigen mit Faible für die Paradoxie und Kalauer zeichnen sein Werk aus. Huberts geht dieser Schreibweise zwar ebenso wenig nach wie Logan, nutzt jedoch McLuhans überbordenden Ideenreichtum, wie es der kanadische Medienforscher selbst vorgeschlagen hat, nämlich als eine Art Werkzeugkasten. Nimmt er doch nur 
ein Konzept heraus und verwendet es in einem ganz anderen Kontext als McLuhan selbst.

Die zweite Fraktion, die Diskursivierer, machen mit McLuhan etwas ganz anderes. An etlichen Beiträgen aus McLuhan neu lesen und Transforming McLuhan ließe sich das zeigen. Um nur einige wenige Beispiele anzuführen: Viele Diskursivierer sind damit beschäftigt, McLuhan zu kritisieren. Beispielsweise wird (wieder einmal) nachgewiesen, welcher problematischen hegelianischen Leitlinie oder welchen messianischen Implikationen McLuhans Kulturund Mediengeschichte folgt (siehe bspw. die Beiträge von Dieter Mersch und Hartmut Winkler in McLuhan neu lesen). Daneben gibt es diejenigen, die McLuhan in, bis dato zumindest, kaum bedachte diskursive Zusammenhänge bringen wollen. Einige erstaunliche Ähnlichkeiten zwischen McLuhan, der Kritischen Theorie und den cultural studies werden in dem Sammelband Transforming McLuhan vorgestellt. Erstaunlich sind diese Ähnlichkeiten vor allem, weil gemeinhin von einer strikten Inkompatibilität zwischen McLuhan und Kritischer Theorie bzw. McLuhan und den cultural studies ausgegangen wurde. Zwischen den affirmativ-fröhlichen Beschreibungen McLuhans und der scharfen Medienkritik aus dem linksorientierten Lager oder auch zwischen McLuhans Vorstellung der Rezipienten als "Servomechanismen» " ${ }^{17}$ ihrer Apparate und der aktiven Rolle, die dem Rezipienten in den Cultural Studies eines Raymond Williams oder John Fiske zugewiesen wird, ist nur schwer zu vermitteln. Diese Vermittlungsarbeit leistet der Band Transforming McLuhan mal sehr gelungen - wenn es beispielsweise um die Ähnlichkeit der Rezeptionsmodelle von McLuhan und den Cultural Studies geht, die jeweils eine Alternative zu einem rein informationstheoretischen Kommunikationsmodell suchen (siehe vor allem den Beitrag von Gary Genosko). Mal ist die Suche nach Übereinstimmungen weniger gelungen. Beispielsweise scheint mir der Versuch nachzuweisen, dass McLuhans «Methode» eigentlich der marxistischen Dialektik entspricht, wie er in den Beiträgen von Donna Flayhan und Paul Gossweiler unternommen wird, ein wenig an den Haaren herbeigezogen und nur um den Preis zu haben, den Begriff Dialektik bis zur Unkenntlichkeit auszudehnen.

Eine dritte Untergruppe der Diskursiverer scheint mir indes sehr viel interessanter zu sein. Es werden hier weder zu kritisierende Diskurspartikel gesucht noch wird über mögliche Ähnlichkeiten zwischen unterschiedlichen Theoriediskursen spekuliert, sondern es wird vielmehr gefragt, wer denn das Beschreibungsvokabular McLuhans aufnahm und nutzte, wie also McLuhans Schriften Einfluss auf andere Akteure gewonnen haben. So hatte er nicht nur immensen Erfolg in der sogenannten ästhetischen Neoavantgarde der 196oer Jahre (siehe dazu die Beiträge von Martina Leeker und Fred Turner in McLuhan neu lesen). Darüber hinaus, so lässt sich in Claus Pias Text aus demselben Band nachlesen, lieferte McLuhan technisch und informationstheoretisch versierten Akteuren Ideen, Metaphern und Szenarien, mit deren Hilfe sie das, was sie am Computer taten, anders und vor allem gesellschaftsrelevant und politisch beschreiben konnten, ohne dass McLuhan selbst viel Ahnung von Computern haben musste: «Das Experimentieren mit Medienfunktionen wird plötzlich als «Medientheorie» artikulierbar, weil McLuhans medientheoretische Diagnose des Computerzeitalters von Leuten gelesen wird, die technisches Verständnis für das Potential des Computers hatten, und nun plötzlich merkten, dass sie es mit einem Medium zu tun haben.» (S. 146)

Hier geht es also zum einen um eine Art Historisierung McLuhans, die über weite Strecken zumindest in der deutschsprachigen Rezeption ein Desiderat war. Zum anderen wird der Focus zugleich verschoben auf die Rolle McLuhans im Kontext künstlerischer und ingenieurwissenschaftlicher Netzwerke. McLuhan wird so nicht einfach als Teil der Ideengeschichte verstanden, sondern vielmehr als Aktant, der innerhalb unterschiedlicher Kontexte funktionalisierbar war.

Den vielen Anhängern der Diskursiver steht die sehr kleine Fraktion der Grammatisierer gegenüber. Sie besteht genau aus zwei Abgeordneten: Zum einen aus dem McLuhan-Biografen W. Terrence Gordon und zum anderen aus dem amerikanischen Kommunikationswissenschaftler John Durham Peters. Gordon wiederholt in seiner unlängst erschienenen Einführung in die Gedankenwelt McLuhans, die den schönen und passenden Untertitel trägt A Guide for the Perplexed, was er bereits in seiner Biografie (und seither immer wieder) über McLuhan geschrieben hat. Erstens: McLuhans CEuvre folge einem roten Faden. Zweitens: Dieser rote Faden ist nicht die Frage nach den Medien, sondern die nach der Grammatik. Peters nimmt Gordons Faden in seinem Beitrag für den Band McLuhan neu lesen auf und liest McLuhan, ausgehend von dessen erstmals 2006 unter dem Titel The Classical Trivium posthum veröffentlichter Doktorarbeit von 1942, ebenfalls als Grammatiker.

«Grammatiken meint in diesem Kontext nicht jemand, der noch den Dativ vom Akkusativ systematisch unterscheiden kann (und will). Vielmehr ist Grammatik 
in antiker Tradition eine der drei klassischen Wege zur Erkenntnis. Die Dialektik wird dabei als eine rationalistische Erkenntnisform verstanden, die Rhetorik primär als Überredungskunst. Von beiden Erkenntniswegen unterscheidet sich die Grammatik fundamental. Peters schreibt dazu: «Die klassischen Grammatiker verstanden Grammatik als Kunst der Interpretation im Allgemeinen, die sich über Literatur hinaus auf das Universum selbst bezog. [...] Grammatiker sind Alchimisten und Enzyklopädisten, die ihren Auftrag darin sehen, alles in einem Bezug auf die grammatikalischen Formen einer zugrunde liegenden sprachlichen Ordnung zu verstehen und zu untersuchen.» (S. 63f.) McLuhan - und das ist für Gordon wie für Peters entscheidend - beschreibe in seiner Dissertation nun nicht nur die Geschichte dieser Grammatiker, sondern setze sich selbst in deren Tradition. Dementsprechend ließen sich auch McLuhans folgende Publikationen als (moderne) Ausgestaltung grammatischer Argumentations- und Erkenntnisformen verstehen.

Ein Grammatiker sucht McLuhan zufolge nach diesen fundamentalen Strukturen in Worten und Dingen vor allem mithilfe von Analogieschlüssen. Dementsprechend gilt: Weniger argumentative Kohärenz und Ableitung sind hier wichtig, keine Klassifikationssysteme werden erstellt. Viel entscheidender ist es, die Verhältnisse, Relationen und eben Ähnlichkeiten von Ding und Wort zu entdecken. Alles wird in Bezug gesetzt zu den übergreifenden grammatikalischen Formen und Strukturen. Buchstäblich Gott und die Welt werden so von McLuhan in Zusammenhang gebracht, auf Ähnlichkeiten hin untersucht und nach universellen grammatikalischen Strukturen durchleuchtet. Das ist ein durch und durch ontologischer, nicht-relativistischer Ansatz und einem vor-modernen Denken in Ähnlichkeiten verpflichtet, wie es Michel Foucault beschrieben hat. ${ }^{18}$ Die Welt zeigt sich, wie sie ist - zumindest dem, der die richtigen Analogien und die geeigneten Darstellungsbzw. Wahrnehmungsweisen findet. McLuhan jedenfalls ist so gesehen tatsächlich niemals modern gewesen. In diesem Zusammenhang versteht McLuhan Medien ana$\log$ (!) zu Metaphern. Sie können zwar helfen, die Welt aus unterschiedlichen Perspektiven in den Blick zu nehmen. Keinesfalls steuern aber die jeweils sherrschenden Nachrichtentechniken alles Verstehen', das wird vielmehr von der vorgängigen Ordnung der Dinge erledigt.

Solch eine sgrammatisierendes Lesart hält, gerade rechtzeitig zu den Feierlichkeiten des hundertsten Geburtstags von McLuhan, einen besonderen Clou bereit, vor allem für

den deutschsprachigen Kontext. Denn der vermeintliche Gründungsvater der Medienwissenschaft hat so gesehen mit Fragen nach Mediendifferenzen, der Materialität der Medien, medialen Aprioris oder der Spezifik «neuer Mediens, also genau den Phänomenen und Erkenntnisweisen, die mit Bezug aufMcLuhan für die Eigenständigkeit des Faches seit den 1980er Jahren reklamiert werden, eigentlich wenig am Hut. Herzlichen Glückwunsch!

1 Siehe dazu: http:/|www.mcluhangalaxy.net|, gesehen am 07.01.11. 2 So sind bspw. zum «Hundertjährigen> bereits zwei Einführungsbändchen angekündigt: Rainer Höltschl, Marshall McLuhan, München (UTB) 2011, und Sven Grampp, Marshall McLuhan - Eine Einführung, Konstanz (UVK) 2011.

3 Wenngleich er diese Rolle auch in der Medienwissenschaft bis dato immer noch spielen darf - siehe bspw. besonders pointiert bei Werner Faulstich, Einführung in die Medienwissenschaft, München (UTB) 2002, S. 22.

4 Siehe dazu etwa: Oliver Lerone Schultz, Marshall McLuhan - Medien als Infrastrukturen und Archetypen, in: Alice Laagay, David Lauer (Hg.), Medientheorien. Eine philosophische Einführung, Frankfurt/M., New York (Campus) 2004, 31-68, v. a. 31.

5 Rainer Leschke, Einführung in die Medientheorie, München (UTB)

2003, 245; Hervorhebung von mir.

6 Jochen Hörisch, Eine Geschichte der Medien. Vom Urknall zum Internet, Frankfurt/M. (Suhrkamp) 2004 (zuerst erschienen unter dem Titel: Der Sinn und die Sinne. Eine Geschichte der Medien, Frankfurt/M. [Eichborn] 2001), 18.

7 Siehe dazu ausführlicher: Geoffrey Winthrop-Young, Friedrich Kittler zur Einführung, Hamburg (Junius) 2005 , 8off. oder auch ders., Kittler in the Anglosphere: "German Media Theory" and other Collateral Damage in Trans-Atlantic Theory Wars, online abrufbar unter: http:// www.mediatrans.ca/Geoffrey_Winthrop.html, gesehen am 10.1.11.

8 Siehe bspw. Friedrich Kittler, Grammophon Film Typewriter, Berlin (Brinkmann \& Bose) 1986, 4 ff.

9 Friedrich Kittler, Optische Medien. Berliner Vorlesung 1999, Berlin (Merve) 2002, $23 \mathrm{f}$.
10 Kittler, Grammophon Film Typewriter, 5 .

11 Kittler, Optische Medien, 23.

12 Unter den hier zu rezensierenden Büchern, ist es vor allem McLuhan neu lesen, in dem dieses Argument schon prominent als wissenschaftshistorische Tatsache in der Einleitung angeführt wird.

13 So etwa im feuilletonistischen Bereich bereits: Jean Améry, Hitler und der Spuknapf. Jean Améry über Marshall McLuhan: Die magischen Kanäle, in: Der Spiegel, 22 (1968), 178-18o.

14 Siehe beispielsweise Leschke, Medientheorie, $245 \mathrm{ff}$., oder auch ausführlicher: Alexander Wittwer, Verwirklichungen. Eine Kritik der Medientheorie, Freiburg (Rombach) 2001, 31ff. Sehr viel früher schon wurde der Vorwurf seitens der Cultural Studies formuliert, prominent von Raymond Williams - siehe bspw. A Structure of Insights (Rezension zu Marshall McLuhans The Gutenberg-Galaxy), in: University of Toronto Quarterly 3/33, 1964, 338-340.

15 Siehe: A Candid Conversation with the High Priest of Popcult and Metaphysican of Media, in: Playboy 3/16 (1969), 53-74, hier 158; auch Online zugänglich unter http://www.digitallantern.net/mc

luhan/mcluhanplayboy.htm, gesehen am 16.01.11.

16 Marshall McLuhan, Die magischen Kanäle. Understanding Media [1964], Dresden, Basel (Verlag der Kunst) 1995, 30.

17 McLuhan, Die magischen Kanäle, 81 .

18 Siehe prominent in: Michel Foucault, Die Ordnung der Dinge. Eine Archäologie der Humanwissenschaften, Frankfurt/M. (Suhrkamp) 1971 (1966), 46ff. 